\title{
Effect of Packing and Storage Conditions on Physical and Physiological Parameters in Extending the Vase Life of Cut Carnation cv. Kiro
}

\author{
P. Pranuthi, T. Suseela, D.V. Swami*, D.R. Salomi Suneetha and V. Sudha Vani
}

Department of Floriculture and Landscape Architecture, Dr. Y.S.R. Horticultural University, Venkataramannagudem, West Godavari dist. (Andhra Pradesh), India

*Corresponding author

\section{A B S T R A C T}

\section{Keywords \\ Carnation, Cold storage, Water uptake, \\ Transpirational loss of \\ water, Water balance and \\ fresh weight change \\ Article Info \\ Accepted: \\ 10 July 2018 \\ Available Online: \\ 10 August 2018}

An experiment was carried out to study the effect of different packing and storage conditions on vase life of cut carnation cv. Kiro. The flowers packed in polypropylene at $5^{\circ} \mathrm{C}$ cold storage under wet condition recorded significantly the highest water uptake $\left(\mathrm{g}\right.$ flower $\left.^{-1}\right)$, whereas significantly the modest transpirational loss of water $\left(\mathrm{g}_{\text {flower }}{ }^{-1}\right)$ resulting in increased water balance $\left(\mathrm{g}_{\text {flower }}{ }^{-1}\right)$ and highest fresh weight change $\left(\mathrm{g}\right.$ flower $\left.{ }^{-1}\right)$ has been recorded by flowers packed in polypropylene at $5^{\circ} \mathrm{C}$ cold storage under wet condition which have extended the vase life period of carnation flower $\mathrm{cv}$. Kiro.

\section{Introduction}

Carnation (Dianthus caryophyllus L.) is an important cut flower in the world. Carnation is in great demand in international as well as domestic markets, therefore it is important to ensure the longest vase life of flowers. Cut flower industry most important aspects is postharvest handling in order to maintain flower freshness and original colour for a long period after cutting from the mother plant for transportation to distant markets to fetch good prices. Two sets of factors are responsible for keeping quality of cut flowers. Internal mechanism that includes balance between water uptake and water loss, stem plugging, respiration rate and production of toxic substances like ethylene. External factors that include environmental conditions and microbial attacks on cut ends.

Post-harvest life of cut flowers also depends upon efficient packaging and storage. An appropriate packaging of cut flowers together with pulsing is helpful to ensure fresh quality of flowers. Packing is a tool for controlling flower quality in the distribution chain. Apart from preventing mechanical damage, the package serves as a barrier between the conditions inside and outside the package. It protects the flowers from unfavorable outside conditions and enables a micro-climate to 
develop inside the package (Lavanya et al., 2016). Packaging must ensure protection of flowers against flower damage, water loss and external conditions, which are detrimental to flowers in transit (Sivaswamy et al., 1999). Storage at low temperature under wet conditions results in low metabolic activities like respiration, transpiration and maintains high humidity and increased cell turgidity and cell enlargement there by keeping the flower quality and increased vase life (Halevy and Mayak, 1981).

\section{Materials and Methods}

The experiment was held at laboratory of Floriculture and Landscape Architecture, College of Horticulture, Dr. Y.S.R. Horticultural University, Venkataramannagudem, West Godavari dist (A.P) during year 2017-18. Experiment laid out in completely randomised design with factorial concept. The total number of treatment combinations are as follows $\mathrm{T}_{1^{-}}$ $\mathrm{P}_{1} \mathrm{~S}_{1}$ : PVC cling film + Ambient temperature $\left(22 \pm 2^{\circ} \mathrm{C}\right) \quad$ (Wet storage), $\mathrm{T}_{2}-\mathrm{P}_{2} \mathrm{~S}_{1}$ : Polyethylene $(100$ gauge $)+$ Ambient temperature $\left(22 \pm 2^{\circ} \mathrm{C}\right)$ (Wet storage), $\mathrm{T}_{3}-\mathrm{P}_{3} \mathrm{~S}_{1}$ : Cellophane paper + Ambient temperature $\left(22 \pm 2^{\circ} \mathrm{C}\right.$ ) (Wet storage), $\mathrm{T}_{4}-\mathrm{P}_{4} \mathrm{~S}_{1}$ : Polypropylene + Ambient temperature $\left(22 \pm 2^{\circ} \mathrm{C}\right)$ (Wet storage), $\mathrm{T}_{5}-\mathrm{P}_{5} \mathrm{~S}_{1}$ : Control (open) + Ambient temperature $\left(22 \pm 2^{\circ} \mathrm{C}\right.$ ) (Wet storage), $\mathrm{T}_{6}-\mathrm{P}_{1} \mathrm{~S}_{2}$ : $\mathrm{PVC}$ cling film + Cold storage of $5^{\circ} \mathrm{C}$ (Wet storage), $\mathrm{T}_{7}-\mathrm{P}_{2} \mathrm{~S}_{2}$ : Polyethylene (100 gauge) + Cold storage of $5^{\circ} \mathrm{C}$ (Wet storage), $\mathrm{T}_{8}-\mathrm{P}_{3} \mathrm{~S}_{2}$ : Cellophane paper + Cold storage of $5^{\circ} \mathrm{C}$ (Wet storage), $\mathrm{T}_{9}-\mathrm{P}_{4} \mathrm{~S}_{2}$ : Polypropylene + Cold storage of $5^{\circ} \mathrm{C}$ (Wet storage), $\mathrm{T}_{10}-\mathrm{P}_{5} \mathrm{~S}_{2}$ : Control (open) + Cold storage of $5^{\circ} \mathrm{C}$ (Wet storage), $\mathrm{T}_{11}-\mathrm{P}_{1} \mathrm{~S}_{3}$ : $\mathrm{PVC}$ cling film + Ambient temperature $\left(22 \pm 2^{\circ} \mathrm{C}\right)$ (Dry storage), $\mathrm{T}_{12}-\mathrm{P}_{2} \mathrm{~S}_{3}$ : Polyethylene (100 gauge) + Ambient temperature $\left(22 \pm 2^{\circ} \mathrm{C}\right)$ (Dry storage), $\quad \mathrm{T}_{13}-\mathrm{P}_{3} \mathrm{~S}_{3}$ : Cellophane paper + Ambient temperature $\left(22 \pm 2^{\circ} \mathrm{C}\right)$ (Dry storage),
$\mathrm{T}_{14}-\mathrm{P}_{4} \mathrm{~S}_{3}: \quad$ Polypropylene + Ambient temperature $\left(22 \pm 2^{\circ} \mathrm{C}\right)$ (Dry storage), $\mathrm{T}_{15}-\mathrm{P}_{5} \mathrm{~S}_{3}$ : Control (open) + Ambient temperature $\left(22 \pm 2^{\circ} \mathrm{C}\right)$ (Dry storage), $\mathrm{T}_{16}-\mathrm{P}_{1} \mathrm{~S}_{4}$ : PVC cling film + Cold storage of $5^{\circ} \mathrm{C}$ (Dry storage), $\mathrm{T}_{17^{-}}$ $\mathrm{P}_{2} \mathrm{~S}_{4}$ : Polyethylene (100 gauge) + Cold storage of $5^{\circ} \mathrm{C}$ (Dry storage), $\mathrm{T}_{18}-\mathrm{P}_{3} \mathrm{~S}_{4}$ : Cellophane paper + Cold storage of $5^{\circ} \mathrm{C}$ (Dry storage), $\mathrm{T}_{19}-\mathrm{P}_{4} \mathrm{~S}_{4}$ : Polypropylene + Cold storage of $5^{\circ} \mathrm{C}$ (Dry storage), $\mathrm{T}_{20}-\mathrm{P}_{5} \mathrm{~S}_{4}$ : Control (open) + Cold storage of $5^{\circ} \mathrm{C}$ (Dry storage). The flowers were kept under dry and wet conditions under both cold storage at $5^{\circ} \mathrm{C}$ as well as ambient temperature $\left(22 \pm 2^{\circ} \mathrm{C}\right)$ by wrapping with different packing materials till the end of vase life period. Water uptake, transpirational loss of water and water balance were observed in wet conditions only i.e. $\mathrm{T}_{1}$ to $\mathrm{T}_{10}$. For physical, physiological, biochemical and microbial studies, same treatments were repeated separately for destructive sampling. Therefore under wet conditions distilled water was used in experimentation. In each conical flask, six flowers were placed and considered as one replication. The treatments were replicated thrice. The individual flower stalks were placed randomly in $500 \mathrm{ml}$ conical flasks containing $300 \mathrm{ml}$ of distilled water under wet conditions. Observations were recorded on water uptake, transpirational loss of water, water balance and fresh weight change.

\section{Results and Discussion}

The water uptake was significant among all the treatment combinations. The highest water uptake $(23.05 \mathrm{~g})$ was recorded in polypropylene packed flowers at $5^{\circ} \mathrm{C}$ cold storage under wet condition $\left(\mathrm{T}_{9}\right)$ and control (without packing) at ambient temperature $\left(22 \pm 2^{\circ} \mathrm{C}\right)$ under wet condition $\left(\mathrm{T}_{5}\right)$ recorded significantly the lowest water uptake (18.34 g) whereas, the remaining all other treatments recorded intermediate values. There were significant differences in water uptake during different days of vase life period. The water 
uptake increased from $2^{\text {nd }}$ day $(20.36 \mathrm{~g})$ to $4^{\text {th }}$ day (20.68 g) and then gradually decreased throughout the vase life period. The interaction effect between treatments and days on water uptake was found significant. The treatment $\mathrm{T}_{9}$, flowers packed in polypropylene at $5^{\circ} \mathrm{C}$ cold storage under wet condition recorded significantly the highest water uptake $(22.96 \mathrm{~g})$ on $2^{\text {nd }}$ day. On $4^{\text {th }}$ day polypropylene packed flowers at $5^{\circ} \mathrm{C}$ cold storage under wet storage $\left(\mathrm{T}_{9}\right)$ recorded the highest water uptake $(23.14 \mathrm{~g})$. Treatment $\mathrm{T}_{9}$ in polypropylene packed flower at $5^{\circ} \mathrm{C}$ cold storage under wet storage recorded the highest water uptake on all the days of vase life study. The increased water uptake with polypropylene packed flowers at cold storage under wet storage might be due positive effect of polypropylene had minimum rate of respiration, transpiration and higher moisture retention and also reduced damage in conducting vessels ensuring continuous water uptake as reported by Waters (1966) and Jeeva and Balakrishnamoorthy (1999) in rose. Storage at low temperature results in low metabolic activities like respiration, transpiration and maintains high humidity also under continuous wet conditions. These results are also in conformity with results of Singh et al., (2009) in gladiolus and Bayleyagne et al., (2012) in rose.

The transpirational loss of water was significant among all the treatment combinations. The lowest transpirational loss of water $(1.07 \mathrm{~g})$ was recorded with flowers packed in polypropylene at $5^{\circ} \mathrm{C}$ cold storage under wet condition $\left(\mathrm{T}_{9}\right)$ and control (without packing) at ambient temperature $\left(22 \pm 2^{\circ} \mathrm{C}\right)$ under wet condition $\left(\mathrm{T}_{5}\right)$ recorded significantly the highest transpirational loss of water $(5.91 \mathrm{~g})$ whereas, the remaining all other treatments recorded intermediate values. There were significant differences in transpirational loss of water during different days of vase life period. The transpirational loss of water significantly increased from $2^{\text {nd }}$ day $(2.79 \mathrm{~g})$ to $4^{\text {th }}$ day $(3.64 \mathrm{~g})$ at each successive interval of observation. The lowest transpirational loss of water was recorded on $2^{\text {nd }}$ day $(2.79 \mathrm{~g})$, whereas, the highest transpirational loss of water $(3.64 \mathrm{~g})$ was recorded on $4^{\text {th }}$ day. The interaction between treatments and days on transpirational loss of water was found significant. The treatment $\left(\mathrm{T}_{9}\right)$ i.e flowers packed in polypropylene at $5^{\circ} \mathrm{C}$ cold storage under wet condition recorded significantly the lowest transpirational loss of water $(0.92 \mathrm{~g})$ on $2^{\text {nd }}$ day. On $4^{\text {th }}$ day, polypropylene packed flowers at cold storage $\left(5^{\circ} \mathrm{C}\right)$ under wet condition $\left(\mathrm{T}_{9}\right)$ recorded the lowest transpirational loss of water $(1.23 \mathrm{~g})$.

Treatment $\left(\mathrm{T}_{9}\right)$ flowers packed in polypropylene at cold storage $\left(5^{\circ} \mathrm{C}\right)$ under wet condition recorded the lowest transpirational loss of water on all the days of vase life study. These findings were also in agreement with the results of Patel and Dhaduk (2010) envisaged that wrapping minimized the transpirational loss of water and deceased permeability for water and $\mathrm{CO}_{2}$, which maintained humidity in wrapping material in tuberose. Similar results were also observed by Sharma et al., (2015) in carnation, Makhwana et al., (2015) in rose and Lavanya et al., (2016) in Jasmine.

The water balance was significant among all the treatment combinations. The highest water balance $(22.01 \mathrm{~g})$ was recorded with flowers packed in polypropylene at $5^{\circ} \mathrm{C}$ cold storage under wet condition $\left(\mathrm{T}_{9}\right)$ and treatment $\left(\mathrm{T}_{5}\right)$ i.e., control (without packing) at ambient temperature $\left(22 \pm 2^{\circ} \mathrm{C}\right)$ under wet condition recorded the lowest water balance $(12.43 \mathrm{~g})$ whereas, the remaining all other treatments recorded intermediate values. There were significant differences in water balance during different days of vase life period. The water balance significantly decreased from $2^{\text {nd }}$ day $(17.58 \mathrm{~g})$ to $4^{\text {th }}$ day $(16.66 \mathrm{~g})$ at each successive interval of observation (Table 1-4). 
Table.1 Effect of different packaging and storage conditions on water uptake $\left(\mathrm{g} \mathrm{flower}^{-1}\right)$ during vase life of carnation (Dianthus caryophyllus L.) cv. Kiro

\begin{tabular}{|c|c|c|c|c|c|c|c|c|c|c|c|c|c|}
\hline \multirow[t]{2}{*}{ Treatments } & & \multicolumn{12}{|c|}{ Days } \\
\hline & 2 & 4 & Mean & 6 & 8 & 10 & 12 & 14 & 16 & 18 & 20 & 22 & 24 \\
\hline $\mathbf{T}_{1}-\mathbf{P}_{1} \mathbf{S}_{1}$ & 19.06 & 19.87 & 19.46 & 18.40 & - & - & - & - & - & - & - & - & - \\
\hline $\mathrm{T}_{2}-\mathbf{P}_{2} \mathrm{~S}_{1}$ & 19.97 & 20.43 & 20.20 & 18.81 & - & - & - & - & - & - & - & - & - \\
\hline $\mathrm{T}_{3}-\mathrm{P}_{3} \mathrm{~S}_{1}$ & 19.75 & 20.20 & 19.97 & 18.73 & - & - & - & - & - & - & - & - & - \\
\hline$\overline{T_{4}-P_{4} S_{1}}$ & 20.04 & 20.84 & 20.44 & 19.67 & 18.83 & - & - & - & - & - & - & - & - \\
\hline $\mathrm{T}_{5}-\mathrm{P}_{5} \mathrm{~S}_{1}$ & 18.56 & 18.12 & 18.34 & - & - & - & - & - & - & - & - & - & - \\
\hline $\mathrm{T}_{6}-\mathrm{P}_{1} \mathrm{~S}_{2}$ & 20.41 & 20.72 & 20.56 & 20.89 & 21.10 & 21.54 & 21.06 & 20.81 & 20.42 & 20.11 & - & - & - \\
\hline$\overline{T_{7}-P_{2} S_{2}}$ & 21.75 & 22.06 & 21.90 & 22.46 & 22.82 & 23.08 & 22.94 & 22.30 & 21.90 & 21.18 & 20.86 & 20.18 & - \\
\hline $\mathrm{T}_{8}-\mathrm{P}_{3} \mathrm{~S}_{2}$ & 20.67 & 20.86 & 20.76 & 21.14 & 21.70 & 22.11 & 21.63 & 21.16 & 20.85 & 20.54 & 20.10 & 19.72 & - \\
\hline $\mathrm{T}_{9}-\mathrm{P}_{4} \mathrm{~S}_{2}$ & 22.96 & 23.14 & 23.05 & 23.95 & 24.10 & 24.92 & 24.30 & 23.96 & 23.23 & 22.95 & 22.37 & 21.90 & 20.27 \\
\hline$\overline{T_{10}-P_{5} S_{2}}$ & 20.43 & 20.64 & 20.53 & 20.77 & 20.91 & 21.02 & 20.85 & 20.11 & 19.79 & 19.51 & - & - & - \\
\hline Mean & 20.36 & 20.68 & & & & & & & & & & & \\
\hline
\end{tabular}

\begin{tabular}{|c|c|c|}
\hline Factors & C.D at 5\% & SE d \\
\hline For treatments (T) & 0.641 & 0.316 \\
\hline For days (D) & 0.286 & 0.141 \\
\hline Factor(T×D) & 0.360 & 0.177 \\
\hline & & \\
\hline *Significant at (P<0.05) & & \\
\hline
\end{tabular}

$\mathrm{P}_{1}$ : PVC cling film

$\mathrm{P}_{2}$ : Polyethylene (100 gauge)

$\mathrm{P}_{3}$ : Cellophane paper

$\mathrm{P}_{4}$ : Polypropylene

$\mathrm{P}_{5}$ : Control (open)

$\mathrm{S}_{1}$ : Ambient temperature $\left(22 \pm 2^{\circ} \mathrm{c}\right)$ (Wet storage)

$\mathrm{S}_{2}$ : Cold storage of $5^{\circ} \mathrm{C}$ (Wet storage) 
Table.2 Effect of different packaging and storage conditions on transpirational loss of water ( $\mathrm{g}$ flower $^{-1}$ ) during vase life of carnation (Dianthus caryophyllus L.) cv. Kiro

\begin{tabular}{|c|c|c|c|c|c|c|c|c|c|c|c|c|c|}
\hline \multirow{2}{*}{ Treatments } & \multicolumn{13}{|c|}{ Days } \\
\hline & 2 & 4 & Mean & 6 & 8 & 10 & 12 & 14 & 16 & 18 & 20 & 22 & 24 \\
\hline $\mathbf{T}_{1}-\mathbf{P}_{1} \mathbf{S}_{1}$ & 4.65 & 5.24 & 4.94 & 6.52 & - & - & - & - & - & - & - & - & - \\
\hline $\mathbf{T}_{2}-\mathbf{P}_{2} \mathbf{S}_{1}$ & 3.42 & 3.970 & 3.69 & 5.61 & - & - & - & - & - & - & - & - & - \\
\hline $\mathrm{T}_{3}-\mathrm{P}_{3} \mathrm{~S}_{1}$ & 4.12 & 4.53 & 4.32 & 5.90 & - & - & - & - & - & - & - & - & - \\
\hline$\overline{\mathrm{T}_{4}-\mathrm{P}_{4} \mathrm{~S}_{1}}$ & 2.98 & 3.45 & 3.21 & 4.94 & 7.11 & - & - & - & - & - & - & - & - \\
\hline $\mathrm{T}_{5}-\mathrm{P}_{5} \mathrm{~S}_{1}$ & 4.96 & 6.86 & 5.91 & - & - & - & - & - & - & - & - & - & - \\
\hline $\mathrm{T}_{6}-\mathrm{P}_{1} \mathrm{~S}_{2}$ & 1.93 & 3.07 & 2.50 & 3.97 & 5.90 & 8.92 & 13.56 & 16.33 & 18.56 & 21.07 & - & - & - \\
\hline $\mathbf{T}_{7}-\mathbf{P}_{2} \mathbf{S}_{2}$ & 1.25 & 2.11 & 1.68 & 2.83 & 4.68 & 7.52 & 12.60 & 15.02 & 17.03 & 22.89 & 24.51 & 26.32 & - \\
\hline $\mathrm{T}_{8}-\mathbf{P}_{3} \mathbf{S}_{2}$ & 1.61 & 2.84 & 2.22 & 3.54 & 5.07 & 8.11 & 12.98 & 15.87 & 17.84 & 22.55 & 25.45 & 28.17 & - \\
\hline $\mathbf{T}_{9}-\mathbf{P}_{4} \mathbf{S}_{2}$ & 0.92 & 1.23 & 1.07 & 1.98 & 3.51 & 6.82 & 11.22 & 14.32 & 16.47 & 20.12 & 23.33 & 25.48 & 28.56 \\
\hline $\mathbf{T}_{10}-\mathbf{P}_{5} \mathbf{S}_{2}$ & 2.10 & 3.14 & 2.62 & 4.50 & 6.71 & 11.50 & 14.70 & 16.93 & 20.10 & 23.26 & - & - & - \\
\hline Mean & 2.79 & 3.64 & & & & & & & & & & & \\
\hline
\end{tabular}

\begin{tabular}{|c|}
\hline Factors \\
\hline For treatments $(\mathbf{T})$ \\
\hline For days $(\mathbf{D})$ \\
\hline Factor(T) $\mathbf{T})$ \\
\hline \\
\hline \\
\hline *Significant at $(\mathbf{P} \leq \mathbf{0 . 0 5})$ \\
\hline
\end{tabular}

\begin{tabular}{|l|l|l|l|}
\hline C.D at 5\% & SE d & $\mathbf{P}_{1}$ : PVC cling film \\
\hline 0.091 & 0.045 & $\mathrm{P}_{2}$ : Polyethylene $(100$ gauge $)$ \\
\hline 0.040 & 0.020 & $\mathrm{P}_{3}$ : Cellophane paper \\
\hline 0.128 & 0.063 & $\mathrm{P}_{4}$ : Polypropylene \\
\hline & & $\mathrm{P}_{5}$ : Control $($ open $)$ \\
\hline & & $\mathrm{S}_{1}$ : Ambient temperature $\left(22 \pm 2^{\circ} \mathrm{c}\right)($ Wet storage $)$ \\
\hline & & $\mathrm{S}_{2}$ : Cold storage of $5^{\circ} \mathrm{C}($ Wet storage $)$ \\
\hline
\end{tabular}


Table.3 Effect of different packaging and storage conditions on water balance $\left(\mathrm{g}_{\text {flower }}{ }^{-1}\right)$ during vase life of carnation (Dianthus caryophyllus L.) cv. Kiro

\begin{tabular}{|c|c|c|c|c|c|c|c|c|c|c|c|c|c|}
\hline \multirow[t]{2}{*}{ Treatments } & \multicolumn{13}{|c|}{ Days } \\
\hline & 2 & 4 & Mean & 6 & 8 & 10 & 12 & 14 & 16 & 18 & 20 & 22 & 24 \\
\hline $\mathbf{T}_{1}-\mathbf{P}_{1} \mathrm{~S}_{1}$ & 14.42 & 13.01 & 13.71 & 11.88 & - & - & - & - & - & - & - & - & - \\
\hline $\mathbf{T}_{2}-\mathbf{P}_{2} \mathbf{S}_{1}$ & 16.56 & 15.45 & 16.00 & 13.20 & - & - & - & - & - & - & - & - & - \\
\hline $\mathbf{T}_{3}-\mathbf{P}_{3} \mathrm{~S}_{1}$ & 15.63 & 14.89 & 15.26 & 12.83 & - & - & - & - & - & - & - & - & - \\
\hline $\mathrm{T}_{4}-\mathrm{P}_{4} \mathrm{~S}_{1}$ & 17.06 & 16.98 & 17.02 & 14.72 & 11.71 & - & - & - & - & - & - & - & - \\
\hline $\mathrm{T}_{5}-\mathrm{P}_{5} \mathrm{~S}_{1}$ & 13.60 & 11.26 & 12.43 & - & - & - & - & - & - & - & - & - & - \\
\hline $\mathrm{T}_{6}-\mathrm{P}_{1} \mathrm{~S}_{2}$ & 18.58 & 17.65 & 18.11 & 16.91 & 15.20 & 12.61 & 11.52 & 10.48 & 9.64 & 9.21 & - & - & - \\
\hline $\mathrm{T}_{7}-\mathbf{P}_{2} \mathrm{~S}_{2}$ & 20.50 & 19.94 & 20.22 & 19.63 & 18.14 & 15.56 & 14.36 & 13.85 & 12.50 & 11.38 & 10.65 & 10.12 & - \\
\hline $\mathrm{T}_{8}-\mathrm{P}_{3} \mathrm{~S}_{2}$ & 19.16 & 18.02 & 18.59 & 17.60 & 16.63 & 13.99 & 13.09 & 12.56 & 10.44 & 10.05 & 9.91 & 9.08 & - \\
\hline $\mathrm{T}_{9}-\mathrm{P}_{4} \mathrm{~S}_{2}$ & 22.05 & 21.97 & 22.01 & 21.17 & 20.59 & 18.10 & 16.89 & 15.79 & 14.63 & 13.69 & 12.54 & 11.30 & 10.50 \\
\hline $\mathrm{T}_{10}-\mathrm{P}_{5} \mathrm{~S}_{2}$ & 18.33 & 17.49 & 17.91 & 16.27 & 14.19 & 9.52 & 9.23 & 9.00 & 8.81 & 8.15 & - & - & - \\
\hline Mean & 17.58 & 16.66 & & & & & & & & & & & \\
\hline
\end{tabular}

\begin{tabular}{|c|}
\hline Factors \\
\hline For treatments (T) \\
\hline For days (D) \\
\hline Factor(T×D) \\
\hline \\
\hline \\
\hline *Significant at $(\mathbf{P} \leq \mathbf{0 . 0 5})$ \\
\hline
\end{tabular}

\begin{tabular}{|c|c|c|}
\hline C.D at 5\% & SE d & \\
\hline 0.581 & 0.286 & \\
\hline 0.260 & 0.128 & \\
\hline 0.821 & 0.405 & \\
\hline & & \\
\hline & & \\
\hline
\end{tabular}

$\mathrm{P}_{1}$ : PVC cling film

$\mathrm{P}_{2}$ : Polyethylene (100 gauge)

$\mathrm{P}_{3}$ : Cellophane paper

$\mathrm{P}_{4}$ : Polypropylene

$\mathrm{P}_{5}$ : Control (open)

$\mathrm{S}_{1}$ : Ambient temperature $\left(22 \pm 2^{\circ} \mathrm{c}\right)$ (Wet storage)

$\mathrm{S}_{2}$ : Cold storage of $5^{\circ} \mathrm{C}$ (Wet storage) 
Table.4 Effect of different packaging and storage conditions on fresh weight change ( flower $\left.^{-1}\right)$ during vase life of carnation (Dianthus caryophyllus L.) cv. Kiro

\begin{tabular}{|c|c|c|c|c|c|c|c|c|c|c|c|c|c|}
\hline \multirow[t]{2}{*}{ Treatments } & \multicolumn{13}{|c|}{ Days } \\
\hline & 2 & 4 & Mean & 6 & 8 & 10 & 12 & 14 & 16 & 18 & 20 & 22 & 24 \\
\hline $\mathbf{T}_{1}-\mathbf{P}_{1} \mathbf{S}_{1}$ & 89.12 & 91.54 & 90.33 & 88.30 & - & - & - & - & - & - & - & - & - \\
\hline $\mathbf{T}_{2}-\mathbf{P}_{2} \mathbf{S}_{1}$ & 93.48 & 96.43 & 94.95 & 92.19 & - & - & - & - & - & - & - & - & - \\
\hline $\mathbf{T}_{3}-\mathbf{P}_{3} \mathbf{S}_{1}$ & 90.41 & 93.50 & 91.95 & 89.63 & - & - & - & - & - & - & - & - & - \\
\hline $\mathbf{T}_{4}-\mathbf{P}_{4} \mathbf{S}_{1}$ & 95.09 & 98.91 & 97.00 & 94.24 & 90.37 & - & - & - & - & - & - & - & - \\
\hline $\mathrm{T}_{5}-\mathrm{P}_{5} \mathrm{~S}_{1}$ & 87.50 & 90.18 & 88.84 & - & - & - & - & - & - & - & - & - & - \\
\hline$T_{6}-P_{1} S_{2}$ & 112.60 & 114.46 & 113.53 & 110.33 & 105.80 & 99.82 & 93.66 & 90.22 & 85.36 & 82.71 & - & - & - \\
\hline $\mathbf{T}_{\mathbf{7}}-\mathbf{P}_{2} \mathbf{S}_{2}$ & 117.33 & 120.58 & 118.95 & 115.27 & 109.45 & 103.68 & 96.58 & 93.42 & 90.75 & 87.48 & 84.90 & 80.93 & - \\
\hline $\mathbf{T}_{8}-\mathbf{P}_{3} \mathbf{S}_{2}$ & 114.36 & 116.76 & 115.56 & 112.27 & 107.36 & 100.78 & 94.13 & 91.68 & 87.49 & 84.69 & 81.26 & 78.98 & - \\
\hline $\mathrm{T}_{9}-\mathrm{P}_{4} \mathrm{~S}_{2}$ & 120.62 & 125.45 & 123.03 & 119.70 & 111.58 & 106.74 & 99.84 & 96.55 & 93.83 & 90.65 & 88.49 & 85.47 & 83.13 \\
\hline $\mathbf{T}_{10}-\mathbf{P}_{5} \mathbf{S}_{2}$ & 110.27 & 112.54 & 111.40 & 108.16 & 104.92 & 98.43 & 91.57 & 88.50 & 84.16 & 80.83 & - & - & - \\
\hline $\mathbf{T}_{11}-\mathbf{P}_{1} \mathbf{S}_{3}$ & 85.08 & - & 42.54 & - & - & - & - & - & - & - & - & - & - \\
\hline $\mathbf{T}_{12}-\mathbf{P}_{2} \mathbf{S}_{3}$ & 86.58 & 81.57 & 84.07 & - & - & - & - & - & - & - & - & - & - \\
\hline $\mathbf{T}_{13}-\mathbf{P}_{3} \mathbf{S}_{3}$ & 85.87 & 80.07 & 82.97 & - & - & - & - & - & - & - & - & - & - \\
\hline $\mathbf{T}_{14}-\mathbf{P}_{4} \mathbf{S}_{3}$ & 87.01 & 84.25 & 85.63 & - & - & - & - & - & - & - & - & - & - \\
\hline $\mathbf{T}_{15}-\mathbf{P}_{5} \mathrm{~S}_{3}$ & 84.34 & - & 42.17 & - & - & - & - & - & - & - & - & - & - \\
\hline $\mathbf{T}_{16}-\mathbf{P}_{1} \mathbf{S}_{4}$ & 97.56 & 95.76 & 96.66 & 93.43 & 91.59 & 88.75 & - & - & - & - & - & - & - \\
\hline $\mathbf{T}_{17}-\mathbf{P}_{2} \mathbf{S}_{4}$ & 105.47 & 101.66 & 103.56 & 98.85 & 96.10 & 93.11 & 86.37 & 83.61 & 80.15 & - & - & - & - \\
\hline $\mathbf{T}_{18}-\mathbf{P}_{3} \mathbf{S}_{4}$ & 101.34 & 98.81 & 100.07 & 96.14 & 93.48 & 90.64 & 85.10 & 80.45 & - & - & - & - & - \\
\hline $\mathbf{T}_{19}-\mathbf{P}_{4} \mathbf{S}_{4}$ & 108.72 & 106.11 & 107.41 & 103.93 & 100.03 & 95.46 & 89.87 & 85.14 & 81.27 & - & - & - & - \\
\hline $\mathrm{T}_{20}-\mathrm{P}_{5} \mathrm{~S}_{4}$ & 95.43 & 92.49 & 93.96 & 90.54 & 88.74 & 85.16 & - & - & - & - & - & - & - \\
\hline Mean & 98.41 & 90.05 & & & & & & & & & & & \\
\hline
\end{tabular}

\begin{tabular}{|c|c|c|c|c|}
\hline Factors & C.D at $5 \%$ & SE d & $\mathbf{P}_{1}$ : PVC cling film & $\mathrm{S}_{1}$ : Ambient temperature $\left(22+2^{\circ} \mathrm{c}\right)$ (Wet storage) \\
\hline For treatments (T) & 3.002 & 1.505 & $\mathrm{P}_{2}$ : Polyethylene (100 gauge) & $\mathrm{S}_{2}$ : Cold storage of $5^{\circ} \mathrm{C}$ (Wet storage) \\
\hline For days (D) & 0.949 & 0.476 & $\mathrm{P}_{3}:$ Cellophane paper & $\mathrm{S}_{3}$ : Ambient temperature $\left(22 \pm 2^{\circ} \mathrm{c}\right)$ (Dry storage) \\
\hline Factor $(T \times D)$ & 4.245 & 2.129 & $\mathrm{P}_{4}$ : Polypropylene & $\mathrm{S}_{4}$ : Cold storage of $5^{\circ} \mathrm{C}$ (Dry storage) \\
\hline & & & $\mathrm{P}_{5}$ : Control (open) & \\
\hline
\end{tabular}


Significantly the highest water balance was recorded on $2^{\text {nd }}$ day $(17.58 \mathrm{~g}$ ) whereas, the lowest water balance (16.66 g) was recorded on $4^{\text {th }}$ day. The interaction between treatments and days on water balance was found significant. The treatment $\left(\mathrm{T}_{9}\right)$ i.e., flowers packed in polypropylene at cold storage $\left(5^{\circ} \mathrm{C}\right)$ under wet condition recorded the highest water balance $(22.05 \mathrm{~g})$ on $2^{\text {nd }}$ day. On $4^{\text {th }}$ day polypropylene packed flowers at cold storage $\left(5^{\circ} \mathrm{C}\right)$ under wet condition $\left(\mathrm{T}_{9}\right)$ recorded the highest water balance $(21.97 \mathrm{~g})$. The treatment $T_{9}$ flowers packed in polypropylene at cold storage $\left(5^{\circ} \mathrm{C}\right)$ under wet condition recorded the highest water balance on all the days of vase life study. Increased water balance in polypropylene packed flowers kept at $5^{\circ} \mathrm{C}$ cold storage under wet condition might be due to the increased water uptake and reduced transpirational loss of water exhibited leading to better maintenance of water balance. These results were in line with the findings of Jitendra kumar et al., (2012) in rose, Jayoti et al., (2014) in tuberose.

The fresh weight change was significant among all the treatment combinations. The highest fresh weight change (123.03 g) was recorded with flowers packed in polypropylene at $5^{\circ} \mathrm{C}$ cold storage under wet condition $\left(\mathrm{T}_{9}\right)$ and control (without packing) at ambient temperature $\left(22 \pm 2^{\circ} \mathrm{C}\right)$ under dry condition $\left(\mathrm{T}_{15}\right)$ recorded significantly the lowest fresh weight change $(42.17 \mathrm{~g})$ whereas, the remaining all other treatments recorded intermediate values.

There were significant differences in fresh weight change during different days of vase life period. The fresh weight change significantly decreased from $2^{\text {nd }}$ day $(98.41 \mathrm{~g}$ ) to $4^{\text {th }}$ day $(90.05 \mathrm{~g})$ at each successive interval of observation. Significantly the highest fresh weight change was recorded on $2^{\text {nd }}$ day $(98.41$ g) whereas, the lowest fresh weight change $(90.05 \mathrm{~g})$ was recorded on $4^{\text {th }}$ day.
The interaction effect between treatments and days on fresh weight change was found significant. The treatment $\left(\mathrm{T}_{9}\right)$ flowers packed in polypropylene at $5^{\circ} \mathrm{C}$ cold storage under wet condition recorded the highest fresh weight change $(120.62 \mathrm{~g})$ on $2^{\text {nd }}$ day. On $4^{\text {th }}$ day polypropylene at $5^{\circ} \mathrm{C}$ cold storage under wet condition $\left(\mathrm{T}_{9}\right)$ recorded the highest fresh weight change $(125.45 \mathrm{~g})$. Treatment $\mathrm{T}_{9}$ flowers packed in polypropylene at $5^{\circ} \mathrm{C}$ cold storage under wet condition recorded the highest fresh weight change on all the days of vase life study. Increased fresh weight change might be due to polypropylene film protects the flower from water loss and permits partial gas exchange that maintain fresh weight and turgidity of flowers. Synergistic effect of low temperature under wet condition also maintains fresh weight of flower. Similar results in accordance with the work of Bayleygn et al., (2012) in rose and Jayoti et al., (2014) in tuberose. The decrease in relative fresh weight with increase in storage period could be associated with a continual loss of water by the flowers through transpiration. The other reason could also be attributed to the fact that decreased capacity of flowers to absorb water from solution or both during storage period. Similar results of reduction in fresh weight at senescence was reported by Jain et al., (2007) in rose and Varu and Barad (2008) in tuberose.

\section{References}

Bayleyegn, A., Tesfye, B. and Workneh, T.S. 2012. Effects of pulsing solution, packaging material and passive refrigeration storage system on vase life and quality of cut rose flowers. African Journal of Biotechnology. 11(16): 38003009.

Halevy, A.H. and Mayak, S. 1981. Senescence and postharvest physiology of cut flowers. Part I. In: Horticultural 
Reviews. Vol 2, AVI Publishing

Westport, conn. Pp. 59-143.

Jain, R., Gupta, Y. C., Bhalla, R. and Thakur, R. 2007b. Effect of wet storage on postharvest quality of rose cv. First Red. Journal of Ornamental Horticulture. 10(4): 260-263.

Jayoti, M., Krishan, P. S., Sellam, P., Babita, S. and Puja, R. 2014. Effect of various chemicals with packaging and storage on Tuberose (Polyanthus tuberose L.) Shelf life. Horticulture Flora Research Spectrum. 3(2): 138-141.

Jeeva, J. L. and Balakrishnamoorthy, G. 1999. Effect of pulsing and packing materials on postharvest life of Rose cv. Happiness. South Indian Horticulture. 47(1-6): 361-363.

Jitendra Kumar., Anis Mirza and Krishan Pal. 2012. Study on Post-Harvest life of cut rose cv. First Red as affected by different chemicals and wrapping materials. Horticulture Flora Research Spectrum. 1(3): 263-266.

Lavanya, V., Nidoni, U. R., Kurubar, A.R., Sharanagouda, H. and Ramachandra, C.T. 2016. Effect of pre-treatment and different packaging materials on shelflife of Jasmine flowers (Jasmine sambac). Environment \& Ecology. 34(1A): 341-345.

Makhwana, R. J., Alka, S., Ahlawat, T. R. and Neelima, P. 2015. Standardization of low temperature storage technology with novel packaging techniques in rose cut flower cv. Passion. Horticultural Flora Research Spectrum. 4(11):44-47.

Patel, D.S. and Dhaduk, B.K. 2010. Efficiency of various wrappings for packaging along with storage temperature and duration on vase life of cut tuberose (Polianthus tuberose L.) cv. Local double. Progressive Horticulture. 42: 143-147.

Sharma, B. P., Dilta, B. S., Dhiman, S. R. and Chaudhary, S. V. S. 2015. Studies on post-harvest life of carnation, (Dianthus caryphyllus L.). International Journal of Farm Sciences. 5(4): 193-201.

Singh, A., Kumar, J. and Kumar, P. 2009. Influence of sucrose pulsing and sucrose in vasse solution on flower quality of modified atmosphere low temperature (MALT)-stored gladiolus cut spikes. Acta Horticulture. 847: 129138.

Sivaswamy. N., Sujatha, A. N., Attri, B. L. and Sharma, T. V. R. S. 1999. Postharvest technology of cut flowers. Agro India. 4: 12-13.

Varu, D.K. and Barad, A.V. 2008 a. Effect of different packing methods on vase life and quality of cut flowers in tuberose (Polyanthus tuberosa L.) cv. Double. Asian Journal of Biological Science. 3(1):159-162.

Waters, W. 1966. Toxicity of certain Florida waters to cut flowers. Proceedings of the Florida State Horticultural Society. 79: 456-459.

\section{How to cite this article:}

Pranuthi, P., T. Suseela, D.V. Swami, D.R. Salomi Suneetha and Sudha Vani, V. 2018. Effect of Packing and Storage Conditions on Physical and Physiological Parameters in Extending the Vase Life of Cut Carnation cv. Kiro. Int.J.Curr.Microbiol.App.Sci. 7(08): 1356-1364. doi: https://doi.org/10.20546/ijcmas.2018.708.154 